\title{
Plasma-based Endogenous Metabolomics Profiling of High-Risk Human Papillomavirus and Their Emerging Roles in the Progression of Cervical Cancer
}

\section{Qin Wang}

Tongren Hospital, Shanghai Jiao Tong University School of Medicine

Min Xu

Tongren Hospital, Shanghai Jiao Tong University School of Medicine

Tingting Chen

Tongren Hospital, Shanghai Jiao Tong University School of Medicine Jing Chen

Tongren Hospital, Shanghai Jiao Tong University School of Medicine Runjie Zhang

Tongren Hospital, Shanghai Jiao Tong University School of Medicine Jin Qiu ( $\sim$ jeanqiuqiu@hotmail.com )

Tongren Hospital, Shanghai Jiao Tong University School of Medicine

\section{Research Article}

Keywords: High-risk human papillomavirus, LC-MS, Ceramides, Metabolomics, Cervical cancer

Posted Date: September 21st, 2021

DOI: https://doi.org/10.21203/rs.3.rs-914686/v1

License: (c) (i) This work is licensed under a Creative Commons Attribution 4.0 International License.

Read Full License 


\section{Abstract}

Objective: High-risk human papillomavirus (HR-HPV) is the main etiological factor for cervical cancer. Accumulating evidence has suggested that the active role of metabolites in the initiation and progression of cancers. This study was to explore the metabolic profiles of HR-HPV infection and their potential functions in cervical cancer.

Methods: Non-targeted metabolomics approach was used to detect metabolic alterations in the plasma obtained from HPV-16 positive (HPV16 (+)), HPV-18 positive (HPV18 (+)) and HPV negative (CTL) individuals, followed by CCK8 experiment to detect the effect of different metabolites on the proliferation of Hela and GH354. A cell migration test then verified significant metabolites on the migration of Hela and GH354. Q RT-qPCR and western blot were used to detect malignant progression related mRNA and protein expression levels of cervical cancer.

Results: HR-HPV groups shared 24 dysregulated metabolites (such as amino acids, ceramides, glycerophosphocholines). Further experiments showed ceramide species, including C8 inhibits cervical cancer cells proliferation and migration in vitro. In contrast, C12 significantly enhanced cervical cancer cells proliferation and migration in vitro. Protein and mRNA expressions indicated C8 and C12 were related to the malignant behavior of cervical cancer in vitro. The underlying mechanism demonstrated that C8 intervention inhibited proliferation and migration in cervical cancer cells via the MAPK/JNK signaling pathway, while $\mathrm{C} 12$ intervention promoted proliferation and migration in cervical cancer cells via the MAPK/ERK signaling pathway. These findings may contribute to the treatment of HR-HPV-induced cervical cancer by intervening in its initiation and progression.

Conclusion: Our study shed some light on how metabolites influenced the relationship between HR-HPV oncogenic capability and metabolic phenotype change and identify species C8 and C12 as critical lipid metabolites that modulate cervical cancer cell's function.

\section{Introduction}

Cervical cancer is the fourth most common female malignant tumor resulting in over 300000 deaths worldwide each year[1, 2]. Cervical cancer is a multifactorial, complex multi-step process related to the activation of proto-oncogenes or the inactivation of tumor suppressor genes. Almost $99.7 \%$ of cervical cancers in women are attributable to human papillomavirus infections[3]. Persistent infection of high-risk human papillomavirus (HR-HPV) is considered as the most vital epidemiologic risk factor for cervical cancer, and HPV types 16 and 18 contribute to over $70 \%$ of cervical cancer[4]. Moreover, HR-HPV leads to the dysfunction of basal epithelial cells and the occurrence of cervical cancer[5].

Tumor cells reprogram their metabolism to support cell growth, proliferation, and differentiation, thus driving cancer progression[6]. Previous studies concluded that metabolites are the final products of various biological processes and maybe the biomarkers reflecting upstream biological events, such as environmental influences and genetic mutations[7]. Metabolomic variations are considered ideal 
biomarkers for the screening and diagnosis of cancers[8]. Metabolites have been defined as biologically active metabolites accompanied by the concept of activity metabolomics[9]. The emerging studies have highlighted the functional role of metabolites in physiology and disease. Also, some examples support mTOR kinase 6 act as active entities in cell nutrients and energy[10]. An additional study unraveled aketoglutarate active macrophage and regulated immunity[11]. Abnormal accumulations of fumarate and succinate, termed oncometabolite, causes potential transformation to malignancy[12]. Metabolites such as lipids, amino acids and bile acids play a role in regulating insulin sensitivity[13]. Lysophosphatidic acid can mediate the cell migration and metastasis of ovarian cancer cells by activating the AMPK pathway[14]. HPV infection drive metabolic modifications and their metabolites as potential markers in predicting the risk of cervical cancer[15]. However, the role of bioactive metabolites and underlying mechanisms remain largely unknown. Metabolomics enables us to identify metabolites with the potential to modulate biological processes associated with cervical cancer caused by HR-HPV infection.

This article aims to explore the difference in the metabolite expression profiles between HR-HPV and negative groups and reveals how metabolites intervene in HR-HPV oncogenic capability. Non-targeted metabolomics was employed to show metabolite expression profiles between HRHPV and negative groups. Interestingly whereas changes observed in cervical cancer cells function due to ceramide species Ceramide-1-Phosphate (d18:1/8:0) and Ceramide-1-Phosphate (d18:1/12:0) interventions result from ceramides action. Thus, this study might provide a prospect of bioactive metabolites in understanding the oncogenic potential of HRHPV.

\section{Materials And Methods}

\subsection{Ethical statement}

All the participants signed an informed consent form, which was approved by the Ethics Committee of Tongren Hospital, Shanghai Jiao Tong University (Shanghai, China).

\subsection{Clinical samples}

A total of 30 individuals (10 HPV16 positives, $10 \mathrm{HPV} 18$ positives and 10 controls) were recruited from July to September 2020. Control (CTL) referred to women without HPV infection. The HPV infected participants' plasma samples were collected at Tongren Hospital, Shanghai, China. The plasma from control groups was from healthy volunteers in the Changning district, Shanghai, China. All participants were aged between 20 and 45 years old and were not pregnant or experiencing menstruation. The sampling was processed by trained gynecologists and followed the approved protocol. All the blood samples were collected in the anticoagulant tubes and centrifuged at $3000 \mathrm{rpm}$ for $15 \mathrm{~min}$ at $4{ }^{\circ} \mathrm{C}$. The supernatant was then transferred into $1.5 \mathrm{ml}$ tubes and stored at $-80{ }^{\circ} \mathrm{C}$ before use.

\subsection{Sample preparation}


After thawing, about $100 \mu$ of the supernatant sample was added into $10 \mu \mathrm{l}$ of 2-chloro-L- phenylalanine $(0.3 \mathrm{mg} / \mathrm{mL})$ and Lyso PC17:0, $0.01 \mathrm{mg} / \mathrm{mL}$ diluted in methanol as internal standard, and then vortexed for 10 s. Pre-cold methanol and acetonitrile $(2 / 1, v / v)$ were mixed and added into the sample, and then vortexed for $1 \mathrm{~min}$, samples were ultrasonically extracted in an ice water bath for $10 \mathrm{~min}$, and held at $-20^{\circ} \mathrm{C}$ for $30 \mathrm{~min}$. After centrifuging at $13000 \mathrm{rpm}$ at $4^{\circ} \mathrm{C}$ for $10 \mathrm{~min}, 300 \mu \mathrm{L}$ of the supernatant was transferred into the LC-MS vial and evaporated to dryness. Next, $400 \mu \mathrm{L}$ of methanol and water $(1 / 4, v / v)$ were applied to each sample, vortexed for $30 \mathrm{~s}$, held at $4^{\circ} \mathrm{C}$ for $2 \mathrm{~min}$, and then stored at $-20^{\circ} \mathrm{C}$. After 30 min, samples were centrifuged at $13000 \mathrm{rpm}$ at $4^{\circ} \mathrm{C}$ for $10 \mathrm{~min}$. The supernatants were aspirated using syringes, filtered through $0.22 \mu \mathrm{m}$ microfilters, transferred to LC vials, and finally stored at $-80^{\circ} \mathrm{C}$.

\subsection{Detection of metabolic profiling by LC-MS}

Metabolic profiles in both electrospray ionization (ESI) positive and ESI negative ion modes were carried out using an ACQUITY UPLC I-Class system (Waters Corporation, Milford, USA) coupled with an AB SCIEX Triple TOF 5600 System (AB SCIEX, Framingham, MA). The binary gradient elution systems consisted of water containing $0.1 \%$ formic acid, $v / v(A)$ and acetonitrile containing $0.1 \%$ formic acid, $v / v,(B)$. The separation was achieved using the following conditions: $20 \%$ B for 2 min; $60 \%$ B for 4 min; $100 \%$ B for 11 min; $100 \%$ B for $13 \mathrm{~min} ; 13.55 \%$ B for $13.5 \mathrm{~min}$ and a final $5 \% \mathrm{~B}$ for $14.5 \mathrm{~min}$. All the samples were analyzed at $4^{\circ} \mathrm{C}$. The injection volume was $2 \mu \mathrm{l}$. In full scan mode ( $\mathrm{m} / \mathrm{z}$ ranges from 70 to 1000$)$ combined with information-dependent acquisition (IDA) mode, data acquisition was performed at a collision energy of $10 \mathrm{eV}(+)$ and $-10 \mathrm{eV}(-)$. Parameters of mass spectrometry were as follows: ion source temperature ranged from $550^{\circ} \mathrm{C}(+)$ to $550^{\circ} \mathrm{C}(-)$ throughout the acquisition; ion spray voltage was

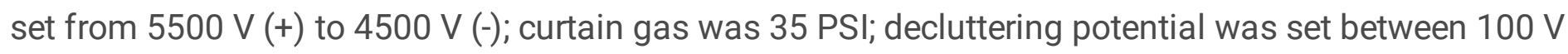
$(+)$ and $-100 \mathrm{~V}(-)$; interface heater temperature was from $550^{\circ} \mathrm{C}(+)$ to $600^{\circ} \mathrm{C} \mathrm{(-).} \mathrm{For} \mathrm{IDA} \mathrm{analysis,} \mathrm{the}$ range of $\mathrm{m} / \mathrm{z}$ was set as $25-1000$, and the collision energy was $30 \mathrm{eV}$.

\subsection{Data analysis}

Metabolites were identified and analyzed by public databases (http://www.hmdb.ca/; http://www.lipidmaps.org/) and self-built databases. The distinct tendency among groups was analyzed using principal component analysis (PCA) and orthogonal partial least-squares discriminant analysis (OPLS-DA) models. The variable importance in projection (VIP) $>1$ and $p$ values $<0.05$ were considered statistically significant. Qualitative and metabolic pathway analyses of differential metabolites were investigated with the Kyoto encyclopedia of genes and genomes (KEGG) online database. Furthermore, the human metabolome database (HMDB) IDs and KEGG IDs of the metabolites were entered into the ingenuity pathway analysis (IPA) server (IPA, Ingenuity ${ }^{\circledR}$ Systems, http://www.ingenuity.com) to analyze networks between metabolites.

\subsection{Ceramides purchase}

C8 Ceramide-1-Phosphate (d18:1/8:0) (\#860532P), C12 Ceramide-1-Phosphate (d18:1/12:0) (\#860531P) were purchased from Sigma-Aldrich (St. Louis, MO, USA). Concentration 50 mM stock solution of C8, C12 
and were prepared in dimethyl sulfoxide (DMSO) and aliquots of this solution were added to cultures, keeping DMSO concentration below $0.1 \%$, same DMSO was added to controls.

\subsection{Cell culture}

Hela (ATCC CCL-2) and GH354 (ATCC CRL-13003) was cultured in DMEM (Dulbecco's modified Eagle's medium) medium, containing 10\% fetal bovine serum (Gibco, New Zealand) and $500 \mu$ penicillinstreptomycin (Gibco, USA), kept in an incubator at $5 \% \mathrm{CO} 2$ and $37^{\circ} \mathrm{C}$. The medium was changed every three days. Cells were digested with $0.05 \%$ trypsin (ScienCell, USA) for inoculation and passage when fused to $80 \%$.

\subsection{CCK8-assay}

The effect of cervical cancer cells proliferation was assayed by CCK8 (TargetMOL, China). Cultured Hela and GH354 cells were suspended in $100 \mu \mathrm{l}$ culture medium with $10 \%$ FBS and inoculated in a 96-well plate (3000 cells/well) along with $0,5,10,20,30 \mu \mathrm{M} \mathrm{C8,C12}$ and $10 \mu \mathrm{l} \mathrm{CCK8}$ solution was added to each well after incubating $24 \mathrm{hrs}, 48 \mathrm{hrs}$ and $72 \mathrm{hrs}$ respectively. The plate was incubated for an additional 1 hour before measuring the absorbance at $450 \mathrm{~nm}$ wavelength using a microplate reader.

\subsection{Cell migration assay}

Cultured cells were suspended in a $2 \mathrm{ml}$ culture medium with $16 \%$ FBS and inoculated in a 6-well plate (100000 cells/well) along with C8 and C12. Control is added with the same DMSO. Migration efficiency was detected at a time of $0 \mathrm{hrs}, 24 \mathrm{hrs}, 36 \mathrm{hrs}$ and $72 \mathrm{hrs}$, respectively.

\subsection{RNA isolation and RT-qPCR analysis}

Total RNA was extracted from cells via TRIzol reagent (Invitrogen, Carlsbad, CA, USA) following manufacturer's instruction, and reverse transcribed using HiScript@囚 QRT SuperMix (Vazyme, Nanjing, China) for reverse transcription-quantitative PCR (RT-qPCR) to obtain cDNA chain. The target genes were amplified in three replicates by the SYBR Green PCR Master Mix (Vazyme, Nanjing, China). The primers were specific for E-cadherin, N-cadherin, Vimentin, MMP9 and GAPDH as follows: E-cadherin primers (Ecadherin F: ATTTTTCCCTCGACACCCGAT; R: TCCCAGGCGTAGACCAAGA), N-cadherin primers (N-cadherin F: AGCCAACCTTAACTGAGGAGT; R: GGCAAGTTGATTGGAGGGATG), Vimentin primers (Vimentin F: TGCCGTTGAAGCTGCTAACTA; R: CCAGAGGGAGTGAATCCAGATTA), MMP9 primers (MMP9 F: AGACCTGGGCAGATTCCAAAC; R: CGGCAAGTCTTCCGAGTAGT), GAPDH primers (GAPDHF: ACAACTTTGGTATCGTGGAAGG; R: GCCATCACGCCACAGTTTC). The RT-qPCR procedures were as follows: $95^{\circ} \mathrm{C}$ for 5 seconds, followed by 40 cycles at $95^{\circ} \mathrm{C}$ for $10 \mathrm{~s}$ and $60^{\circ} \mathrm{C}$ for $30 \mathrm{~s}$. Quantified mRNA was normalized to beta-actin as a control. The relative expression of mRNA was determined by the 2$\triangle \triangle \mathrm{CT}$ method.

\subsection{Protein extraction and western blot analysis}


Cells were prepared by homogenization in the presence of protease inhibitors (Thermo Fisher Scientific, Waltham, MA, USA), and centrifuged to remove cell pellet. The samples were heat-denatured at $95^{\circ} \mathrm{C}$ for 5 minutes with $5 \times$ SDS-PAGE loading buffer and fractionated on 10\% SDS-PAGE gels (Bio-Rad, Hercules, CA, USA). GAPDH (HRP-60004, Proteintech, Manchester, UK) was used as a standard control protein. Following electrophoresis, proteins were transferred to a nitrocellulose membrane and blocked with $5 \%$ skim milk powder. Primary antibodies used Anti-E-cadherin, Anti-N-cadherin, Anti-MMP9 and AntiVimentin antibodies (1;2000, Proteintech, Manchester, UK).

\subsection{Statistical analysis}

Univariate analysis of variance (ANOVA) quantified the differences between the HR-HPV infected and the CTL groups with GraphPad Prism 6.0. Data were presented as the mean \pm standard error (SE). P-values were determined using a two-tailed Student's t-test or one-way analysis of variance (ANOVA) with a Tukey's post hoc correction for multiple group comparisons. All data analyses were processed using GraphPad Prism, version 5.0 (GraphPad Software, San Diego, CA). A two-sided $P$-value of $<0.05$ was considered statistically significant. Significance was set as ${ }^{\star} p<0.05, * \star p<0.01$, $* \star \star p<0.001$, and ${ }^{\star \star \star \star} p<$ 0.0001 .

\section{Results}

\subsection{HR-HPV infection accompanied by distinct metabolome profiles}

To examine the differences in CTL and HR-HPV infection group metabolites, we conducted a multivariate assessment and OPLS-DA analysis. After the PCA model was established, they plot a separation tendency between the CTL group and the HPV16 (+) (Fig. 1A) or HPV18 (+) group (Fig. 1B). OPLS-DA models were obtained with principal predictive components and principal orthogonal components. OPLSDA removed unassociated data from the dataset and then verified the metabolic profiles' dissolution between groups (Fig. 1C-D). We next performed a permutation examination of the OPLS-DA model. The R2 and Q2 intercept values were $(0.0,0.893)$ and $(0.0,-0.285)$ respectively between CTL and HPV16 $(+)$ infection group (Fig. 1E), and (0.0,0.862), $(0.0,-0.321)$ respectively between CTL and HPV18 (+) infection group (Fig. 1F).

\subsection{Identification of differential metabolites in HR-HPV infection and CTL plasma}

A total of 88 differential metabolites in the HPV16 (+) group (VIP $>1, p<0.05)$ were identified, among which 31 metabolites were significantly up-regulated and 57 metabolites were significantly downregulated (Fig. 2A). In addition, a total of differentially expressed 101 metabolites were identified in the HPV18 (+) group, among which 26 metabolites were significantly up-regulated and 75 metabolites were significantly down-regulated (Fig. 2B). Further pathway analysis $(p<0.05)$ revealed that most enriched metabolic pathways were almost in the high-risk groups (Fig. 2C-D), indicating that HPV16 and HPV18 may have similar metabolic functions in the initiation and progression of cervical cancer. To evaluate similarities and dissimilarities in the datasets, we compared the number of shared and unique 
metabolites among the groups. Venn diagram showed 24 identified metabolites (Fig. 2E). Details of the metabolites with the highest difference were summarized in Table 1. Compared to the CTL group, the HRHPV groups shared 12 up-regulated metabolites and 12 down-regulated metabolites respectively. Metabolites were roughly categorized into twelve distinct classes (Fig. 2F). Amino acids and peptides (Fig. 3A) and monosaccharides (Fig. 3B) were reduced in HRHPV groups compared to the CTL group, while glycerophosphocholines were increased and D-Mannitol was decreased in HR-HPV groups (Fig. 3C). Steroid conjugates and benzene derivatives were increased, while ceramides were reduced in HR-HPV groups (Fig. 3D-F). Additionally, two acids with six and five carbon atoms in their fatty acid moieties were detected.

\section{3 $\mathrm{C} 8$ and $\mathrm{C} 12$ regulate the proliferation and migration of $\mathrm{CC}$ cell lines and malignant behavior related molecules at mRNA and protein levels}

We conducted the assays to examine the proliferation and migration potency of the ceramides compounds on cervical cancer cell lines Hela and GH354, it turned out the proliferation rate of $\mathrm{C} 8$ intervention on HeLa and GH354 cells was declined while C12 intervention on HeLa and GH354 cells was restored based on CCK8 assay results (Fig. 4A-D). Comparing the migration index of C8 and C12 intervention cell lines on 0 hour and 48 hours revealed that migration index was significantly diminished in the C8 group but increased for C12 intervention (Fig. 4E-F). To verify the observed effects of ceramide species intervention in vivo, we analyzed the expression levels of several molecules closely related to malignant behavior by western blotting and Q PCR experiments. Q PCR analysis revealed the changes in E-cadherin, N-cadherin, Vimentin and MMP9 expression on C8 (concentration is $30 \mu \mathrm{M}$ ) and $\mathrm{C} 12$ (concentration is $10 \mu \mathrm{M}$ ) intervention on HeLa and GH354 cells. Specifically, E-cadherin was up-regulated after $\mathrm{C} 8$ intervention on Hela and GH354. Conversely, N-cadherin, Vimentin were down-regulated after C8 intervention on Hela (Fig. 4G, I). N-cadherin and MMP9 were up-regulated after C12 intervention in Hela and GH354 (Fig. $4 \mathrm{H}, \mathbf{J}$ ). Western blot verified that significant upregulated E-cadherin and downregulated $\mathrm{N}$-cadherin after $\mathrm{C} 8$ intervention on Hela and GH354 at the protein level, and opposite trend after $\mathrm{C} 12$ intervention (Fig. 5B-C).

\subsection{Interaction network and downstream effects of differential metabolites}

Dysregulated metabolites were imported into IPA software for further biological pathway prediction to reveal potential targets and mechanisms, and its results indicated a close association with PI3K/AKT signaling, mTOR signaling and PTEN signaling, and specific lipids were associated with ERK/MAPK signaling, TGF- $\beta$ signaling and PLA2G2A regulation (Fig. 5A). Thus, whether $\mathrm{C} 8$ and $\mathrm{C} 12$ intervention modified the expression of molecules in the signaling pathway was then explored with Western blot analysis, and it turned out that the expression levels of JNK remained the same regardless of C8 intervention, but the expression level of Bax was up-regulated whereas the expression level of P-JNK and Bcl2 were down-regulated after C8 intervention, which thereby inhibited the activity of MAPK (Fig. 5B).

The expression levels of ERK remained the same regardless of C12 intervention, and the expression level 
of Bax was down-regulated, whereas the expression levels of P-ERK and Blc2 were up-regulated after C12 intervention (Fig. 5C).

\section{Discussion}

HR-HPV is one of the potent human carcinogens. Persistent HRHPV infection is a necessary risk factor for the cervix and cervical cancer[16]. It induces epithelial cells' malignant transformation and suppresses the immune response via encoding oncoproteins[17]. For instance, E6 and E7 proteins encoded by HRHPV could promote cervical cancer[18, 19], E6 protein encourages the growth of cervical cancer cells by targeting P53 protein[20], and E7 protein immortalizes human epithelial cells by targeting pRb protein[21]. Rapid proliferation is a driving force for the massive energy required by malignant cells to adapt to metabolic modifications[22]. Driven by oncoproteins, metabolites delineate a comprehensive characterization of HR-HPV's molecular mechanisms[22]. Besides, the abnormal expression or activation of metabolic pathway-related enzymes is tightly associated with the occurrence of cancers[23]. Previous studies revealed that E6/E7 could also regulate the glycolytic pathway via elevated expression of hexokinase-II, acting as a promotor in HPV-associated cervical lesions in serum [24]. However, the metabolic profile in response to HPV16/18 infection in plasma has not yet been elucidated. Thus, metabolomics analysis may be helpful to explore HRHPV infection and its carcinogenic effects.

In our study, metabolomics analysis was applied to HPV16 (+), HPV18 (+) and CTL groups. The two HRHPV groups shared 12 up-regulated metabolites and 12 down-regulated metabolites compared to the control. These metabolites were mainly divided into 6 categories, and they are amino acids and peptides, ceramides, fatty acids, glycerophosphocholines, monosaccharides and steroid conjugates. Notably, LysoPC (P-16:0) and PC (0-10:1(9E)/0:0) were increased in both HPV16(+) and HPV18(+) groups in our study indicating that lysoPC and PC might play a role in HR-HPV-induced oncogenesis.

Phosphatidylcholine (PC) and lysophosphatidylcholine (LysoPC) were previously considered as potential biomarkers for cervical cancer[25]. LysoPC originates from the cleavage of PC, the main component of oxidatively damaged low-density lipoprotein, which was reported can aggregate inflammation and plays an important role in the invasion, metastasis and prognosis of tumors[16]. In addition, an increasing body of evience has indicated that enzymes that participate in key lipid metabolism are potential therapeutic targets, as they either inhibit their synthesis or stimulate their degradation[9]. Lactate dehydrogenase $A$ is

a key enzyme for lactic acid synthesis, and it also could promote apoptosis and leads to a decrease in the cell cycle when it is inhibited[26]. Phosphoglycerate dehydrogenase, the key enzyme of serine biosynthesis, its inhibition has potential therapeutic value in lung adenocarcinoma[12]. Interestingly, we observed an opposite trend of Ceramide 1-Phosphate (CerP) (d18:1/18:0), it was significantly decreased in both HPV16(+) and HPV18(+) groups, Ceramide is a component of eukaryotic cell membranes that acts as a bioactive lipid in apoptosis, inflammation, cell cycle arrest and the heat shock response[13]. Recent papers found that Ceramide synthase 2-C -ceramide axis limits the metastatic potential of ovarian cancer cells[27], and ceramide in breast cancer patients was correlated with prognostic[28]. Studies have reported that CerP lipidoids could regulate cell proliferation, apoptosis and migration[29], implying that CerP might play a role in HRHPV-induced oncogenesis, however, CerP lipidoids function has not been 
reported yet in CC, and our results suggested that C8 and C12 have an important influence on cervical cancer cell proliferation and migration in vitro. The mRNA and protein expression levels of the malignant indicators for cervical cancer helped to verify the function of ceramide in vitro.

Metabolites serve as controllers of biological processes and phenotype[9], and tumorigenesis may change the overall metabolism of the human body. Therefore, the changes in these biological processes are reflected in the metabolomics, including amino acids and lipid metabolites[30]. Bioactive metabolome drives phenotype modulation by participating in life activities' regulation process and exerting their biological activities through various pathways such as competitive inhibition, post-translational modifications, and signal transduction[31]. Accumulation of oncometabolite is a causal process in malignant conversion that contribute to propagating cancer.

Our study revealed that the dysregulated metabolites were closely associated with PI3K/AKT signaling, mTOR signaling and specific lipids were associated with ERK/MAPK signaling, TGF- $\beta$ signaling and PLA2G2A regulation. The PI3K/AKT/mTOR network plays a key role in HRHPV oncogenes in the host cell[32], and the abnormal activation of PI3K/mTORC2/ AKT signaling pathway could increase migration of keratinocytes and eventually lead to carcinogenesis[33, 34]. It is suggested that ERK-mediated phosphorylation is related to sphingosine metabolism and subsequent inflammation[35], which is an important target for cervical cancer[36]. Besides, the AMPK signaling pathway also plays a vital role in cervical cancer and is closely related to the malignant progression of cervical cancer[37]. The IPA analysis indicated that HRHPV-induced dysregulated metabolites were closely associated with cervical cancer risk. To directly examine the possible mechanism underlying the observed phenotypic changes, we analyzed the expression levels of molecules in the signaling pathway and found that C8 intervention inhibited proliferation and migration in cervical cancer cells via the MAPK/JNK signaling pathway, while C12 intervention promoted proliferation and migration in cervical cancer cells via the MAPK/ERK signaling pathway. Our study provides preliminary evidence that metabolites link the relation between tumorigenesis and metabolic phenotype changes.

In summary, HR-HPV infection-induced distinct metabolites profile changes in humans. The altered metabolites may contribute to the process of HPV16/18 infection and cervical cancer. This paper is the first to identify $\mathrm{C} 8$ and $\mathrm{C} 12$ as critical lipid metabolites that modulate cervical cancer cell's function. Our study provides novel insights into the mechanism of the oncogenic process of HPV16/18 infection.

\section{Declarations}

\section{Conflict of Interest}

The authors declare that they have no competing interests.

\section{Author Contribution}


QW and MX have drafted the manuscript and revised it critically for important intellectual content, and they both conducted the experiments in this study.

TC and JC have made substantial contribution to acquisition and analysis of data.

RZ and JQ have designed and funded this study. Also, they have given final approval of this study.

\section{Funding}

Project supported by National Natural Science Foundation of China (No. 81903006) and Changning District Science and Technology Commission (No. CNKW2018Y06)

\section{Acknowledgments}

Project supported by National Natural Science Foundation of China (No. 81903006) and Changning District Science and Technology Commission (No. CNKW2018Y06).

\section{References}

1. Bray, F., Ferlay, J., Soerjomataram, I., Siegel, R., Torre, L. \& Jemal, A. (2018) Global cancer statistics 2018: GLOBOCAN estimates of incidence and mortality worldwide for 36 cancers in 185 countries, CA: a cancer journal for clinicians. 68, 394-424.

2. Cohen, P., Jhingran, A., Oaknin, A. \& Denny, L. (2019) Cervical cancer, Lancet (London, England). 393, 169-182.

3. Walboomers, J., Jacobs, M., Manos, M., Bosch, F., Kummer, J., Shah, K., Snijders, P., Peto, J., Meijer, C. \& Muñoz, N. (1999) Human papillomavirus is a necessary cause of invasive cervical cancer worldwide, The Journal of pathology. 189, 12-9.

4. Wang, R., Pan, W., Jin, L., Huang, W., Li, Y., Wu, D., Gao, C., Ma, D. \& Liao, S. (2020) Human papillomavirus vaccine against cervical cancer: Opportunity and challenge, Cancer letters. 471, 88102.

5. Kuehn, B. (2021) WHO Launches Global Push to Eliminate Cervical Cancer, JAMA. 325, 213.

6. Sun, C., Li, T., Song, X., Huang, L., Zang, Q., Xu, J., Bi, N., Jiao, G., Hao, Y., Chen, Y., Zhang, R., Luo, Z., Li, X., Wang, L., Wang, Z., Song, Y., He, J. \& Abliz, Z. (2019) Spatially resolved metabolomics to discover tumor-associated metabolic alterations, Proceedings of the National Academy of Sciences of the United States of America. 116, 52-57.

7. Nicholson, J. \& Lindon, J. (2008) Systems biology: Metabonomics, Nature. 455, 1054-6.

8. Cheung, P., Ma, M., Tse, H., Yeung, K., Tsang, H., Chu, M., Kan, C., Cho, W., Ng, L., Chan, L. \& Wong, S. (2019) The applications of metabolomics in the molecular diagnostics of cancer, Expert review of molecular diagnostics. 19, 785-793.

9. Rinschen, M., Ivanisevic, J., Giera, M. \& Siuzdak, G. (2019) Identification of bioactive metabolites using activity metabolomics, Nature reviews Molecular cell biology. 20, 353-367. 
10. Zoncu, R., Efeyan, A. \& Sabatini, D. (2011) mTOR: from growth signal integration to cancer, diabetes and ageing, Nature reviews Molecular cell biology. 12, 21-35.

11. Liu, P., Wang, H., Li, X., Chao, T., Teav, T., Christen, S., Di Conza, G., Cheng, W., Chou, C., Vavakova, M., Muret, C., Debackere, K., Mazzone, M., Huang, H., Fendt, S., Ivanisevic, J. \& Ho, P. (2017) aketoglutarate orchestrates macrophage activation through metabolic and epigenetic reprogramming, Nature immunology. 18, 985-994.

12. Yang, M., Soga, T. \& Pollard, P. (2013) Oncometabolites: linking altered metabolism with cancer, The Journal of clinical investigation. 123, 3652-8.

13. Yang, Q., Vijayakumar, A. \& Kahn, B. (2018) Metabolites as regulators of insulin sensitivity and metabolism, Nature reviews Molecular cell biology. 19, 654-672.

14. Kim, E., Park, J., Lim, S., Choi, J., Kim, H., Seok, H., Seo, J., Oh, K., Lee, D., Kim, K., Ryu, S. \& Suh, P. (2011) Activation of AMP-activated protein kinase is essential for lysophosphatidic acid-induced cell migration in ovarian cancer cells, The Journal of biological chemistry. 286, 24036-45.

15. Godoy-Vitorino, F., Ortiz-Morales, G., Romaguera, J., Sanchez, M., Martinez-Ferrer, M. \& Chorna, N. (2018) Discriminating high-risk cervical Human Papilloma Virus infections with urinary biomarkers via non-targeted GC-MS-based metabolomics, PloS one. 13, e0209936.

16. Qiu, F., Su, B., Li, Z., Chen, W., Cao, L., Chen, F., Liu, D., He, J. \& Lin, N. (2019) New serum biomarker identification and analysis by mass spectrometry in cervical precancerous lesion and acute cervicitis in South China, Cancer management and research. 11, 6151-6162.

17. Abudula, A., Rouzi, N., Xu, L., Yang, Y. \& Hasimu, A. (2020) Tissue-based metabolomics reveals potential biomarkers for cervical carcinoma and HPV infection, Bosnian journal of basic medical sciences. 20, 78-87.

18. Crosbie, E., Einstein, M., Franceschi, S. \& Kitchener, H. (2013) Human papillomavirus and cervical cancer, Lancet (London, England). 382, 889-99.

19. Ford, M., Cannady, K., Nahhas, G., Knight, K., Chavis, C., Crawford, B., Malek, A., Martino, E., Frazier, S., Gathers, A., Lawton, C., Cartmell, K. \& Luque, J. (2020) Assessing an intervention to increase knowledge related to cervical cancer and the HPV vaccine, Advances in cancer research. 146, 115137.

20. Xu, L., Selk, A., Garland, S., Bogliatto, F., Kyrgiou, M., Weyers, S. \& Arbyn, M. (2019) Prophylactic vaccination against human papillomaviruses to prevent vulval and vaginal cancer and their precursors, Expert review of vaccines. 18, 1157-1166.

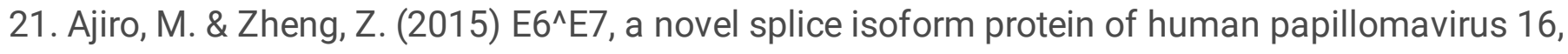
stabilizes viral E6 and E7 oncoproteins via HSP90 and GRP78, mBio. 6, e02068-14.

22. Müller-Schiffmann, A., Beckmann, J. \& Steger, G. (2006) The E6 protein of the cutaneous human papillomavirus type 8 can stimulate the viral early and late promoters by distinct mechanisms, Journal of virology. 80, 8718-28.

23. Zhang, B., Chen, W. \& Roman, A. (2006) The E7 proteins of low- and high-risk human papillomaviruses share the ability to target the pRB family member p130 for degradation, 
Proceedings of the National Academy of Sciences of the United States of America. 103, 437-42.

24. Nilsson, R., Jain, M., Madhusudhan, N., Sheppard, N., Strittmatter, L., Kampf, C., Huang, J., Asplund, A. \& Mootha, V. (2014) Metabolic enzyme expression highlights a key role for MTHFD2 and the mitochondrial folate pathway in cancer, Nature communications. 5, 3128.

25. Zeng, Q., Chen, J., Li, Y., Werle, K., Zhao, R., Quan, C., Wang, Y., Zhai, Y., Wang, J., Youssef, M., Cui, R., Liang, J., Genovese, N., Chow, L., Li, Y. \& Xu, Z. (2017) LKB1 inhibits HPV-associated cancer progression by targeting cellular metabolism, Oncogene. 36, 1245-1255.

26. Thompson, C. (2009) Metabolic enzymes as oncogenes or tumor suppressors, The New England journal of medicine. $360,813-5$.

27. Zhang, X., Sakamoto, W., Canals, D., Ishibashi, M., Matsuda, M., Nishida, K., Toyoshima, M., Shigeta, S., Taniguchi, M., Senkal, C., Okazaki, T., Yaegashi, N., Hannun, Y., Nabe, T. \& Kitatani, K. (2021) Ceramide synthase 2-C -ceramide axis limits the metastatic potential of ovarian cancer cells, FASEB journal : official publication of the Federation of American Societies for Experimental Biology. 35, e21287.

28. Moro, K., Nagahashi, M., Gabriel, E., Takabe, K. \& Wakai, T. (2019) Clinical application of ceramide in cancer treatment, Breast cancer (Tokyo, Japan). 26, 407-415.

29. Gomez-Muñoz, A., Presa, N., Gomez-Larrauri, A., Rivera, I., Trueba, M. \& Ordoñez, M. (2016) Control of inflammatory responses by ceramide, sphingosine 1-phosphate and ceramide 1-phosphate, Progress in lipid research. 61, 51-62.

30. Liu, P., Zhu, W., Chen, C., Yan, B., Zhu, L., Chen, X. \& Peng, C. (2020) The mechanisms of lysophosphatidylcholine in the development of diseases, Life sciences. 247, 117443.

31. Rivera, I., Ordoñez, M., Presa, N., Gangoiti, P., Gomez-Larrauri, A., Trueba, M., Fox, T., Kester, M. \& Gomez-Muñoz, A. (2016) Ceramide 1-phosphate regulates cell migration and invasion of human pancreatic cancer cells, Biochemical pharmacology. 102, 107-119.

32. Dong, J., Lei, H., Liang, Q., Tang, Y., Zhou, Y., Wang, Y., Zhang, S., Li, W., Tong, Y., Zhuang, G., Zhang, L., Chen, H., Zhu, L. \& Shen, Y. (2018) Overcoming erlotinib resistance in EGFR mutation-positive lung adenocarcinomas through repression of phosphoglycerate dehydrogenase, Theranostics. 8, 18081823.

33. Tango, C., Seo, S., Kwon, M., Lee, D., Chang, H. \& Kim, M. (2020) Taxonomic and Functional Differences in Cervical Microbiome Associated with Cervical Cancer Development, Scientific reports. 10, 9720.

34. Bossler, F., Hoppe-Seyler, K. \& Hoppe-Seyler, F. (2019) PI3K/AKT/mTOR Signaling Regulates the Virus/Host Cell Crosstalk in HPV-Positive Cervical Cancer Cells, International journal of molecular sciences. 20.

35. Charette, S. \& McCance, D. (2007) The E7 protein from human papillomavirus type 16 enhances keratinocyte migration in an Akt-dependent manner, Oncogene. 26, 7386-90.

36. Spiegel, S. \& Milstien, S. (2011) The outs and the ins of sphingosine-1-phosphate in immunity, Nature reviews Immunology. 11, 403-15. 
37. Singh, S., Upadhyay, A., Ajay, A. \& Bhat, M. (2007) p53 regulates ERK activation in carboplatin induced apoptosis in cervical carcinoma: a novel target of p53 in apoptosis, FEBS letters. 581, 28995.

\section{Figures}
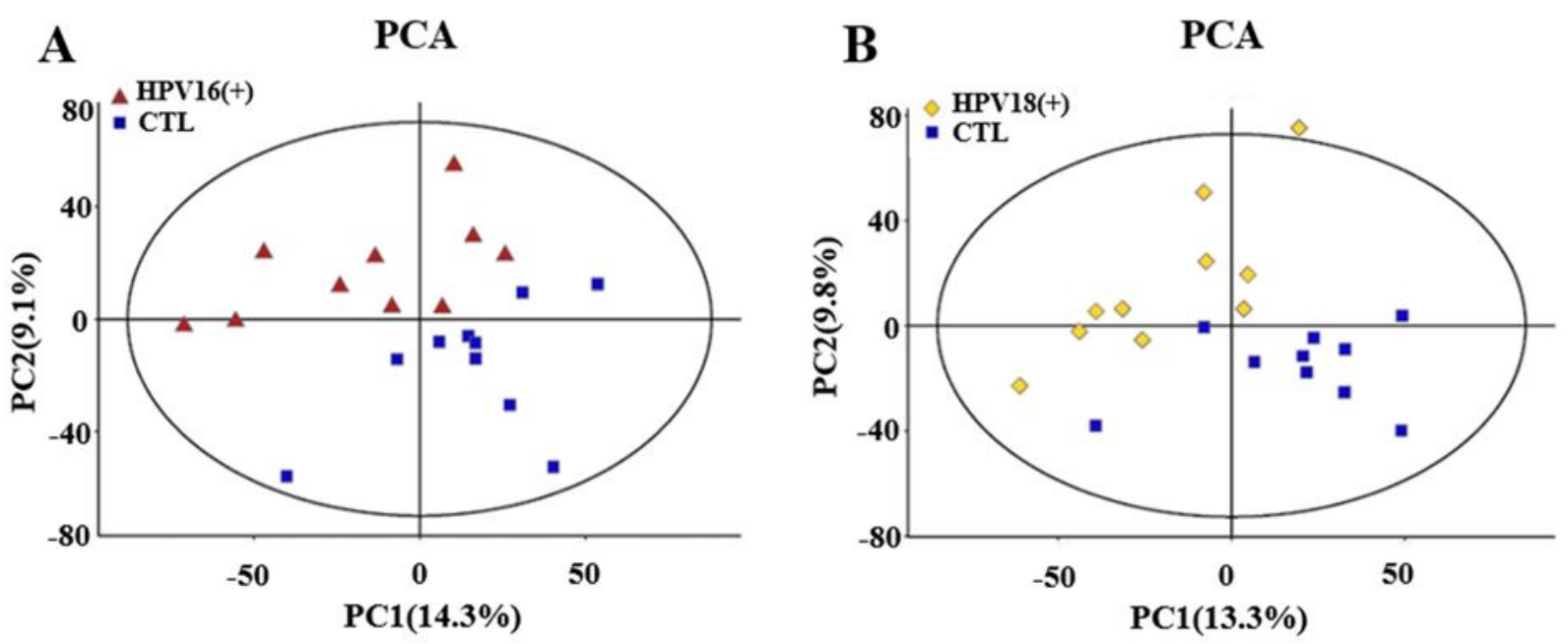

C
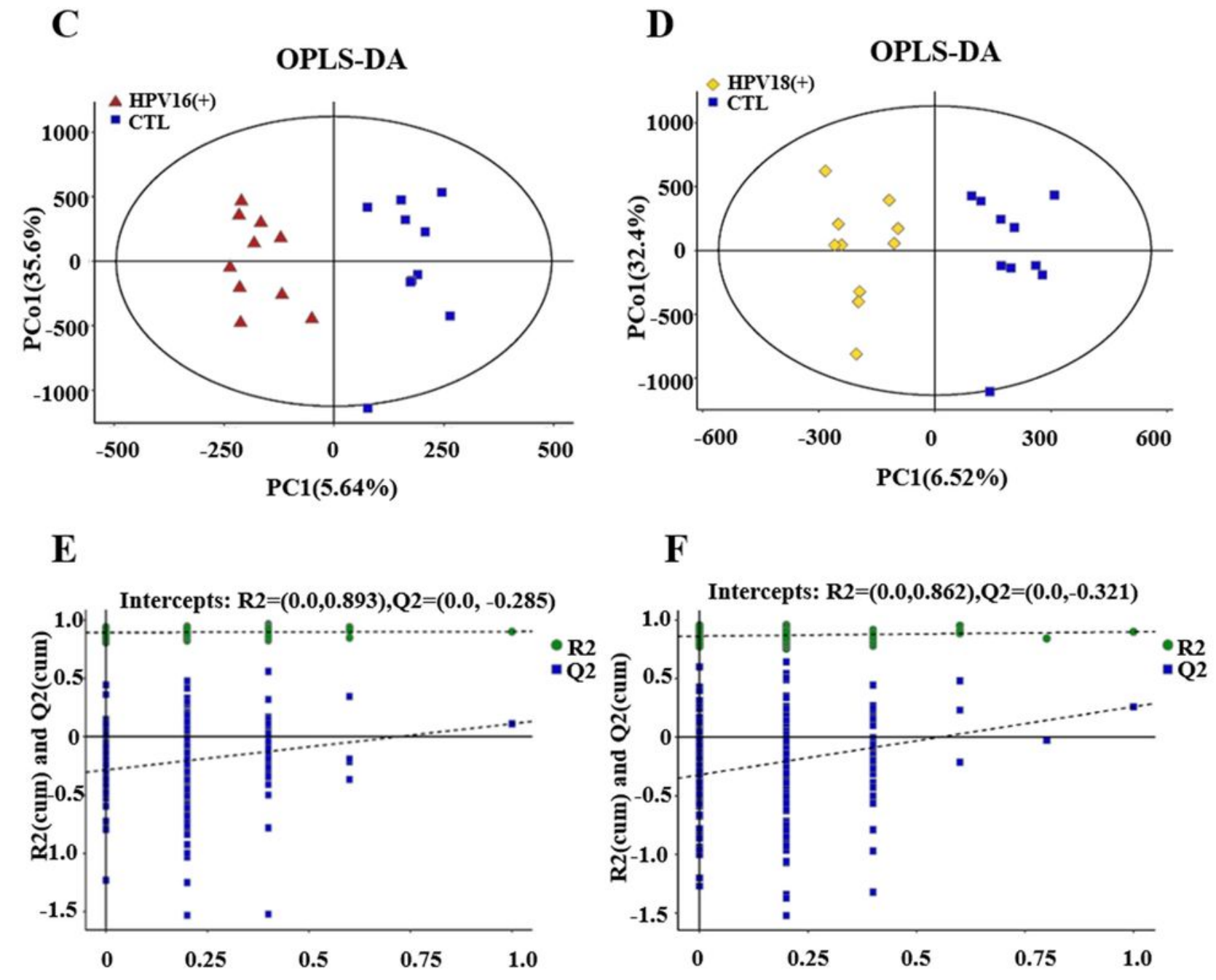

Figure 1 
PCA score plots discriminating the metabolic profiles between CTL and HRHPV infection groups and permutation test of OPLS-DA models. A. Principal component analysis (PCA) showed a distinct metabolome profile in HPV16 (+) groups compared to the CTL group. X-axis and Y-axis represent the first and second principal components, respectively. B. PCA showed a distinct metabolome profile in HPV18 (+) groups compared to the CTL group. C, D. Statistical validation with permutation analysis (200 times) of the corresponding OPLS-DA model of HPV16 (+), HPV18 (+) and CTL. E. Permutation tests were obtained from LC-MS/MS data of HPV16 (+) and CTL F. Permutation tests were obtained from LCMS/MS data of HPV18 (+) and CTL. The intercept values of the regression line and the Y-axis are R2 and Q2, respectively. 


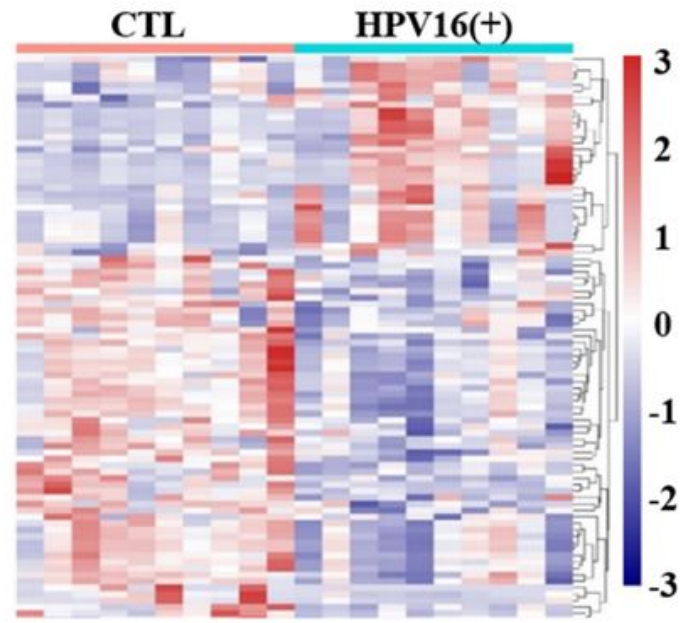

C

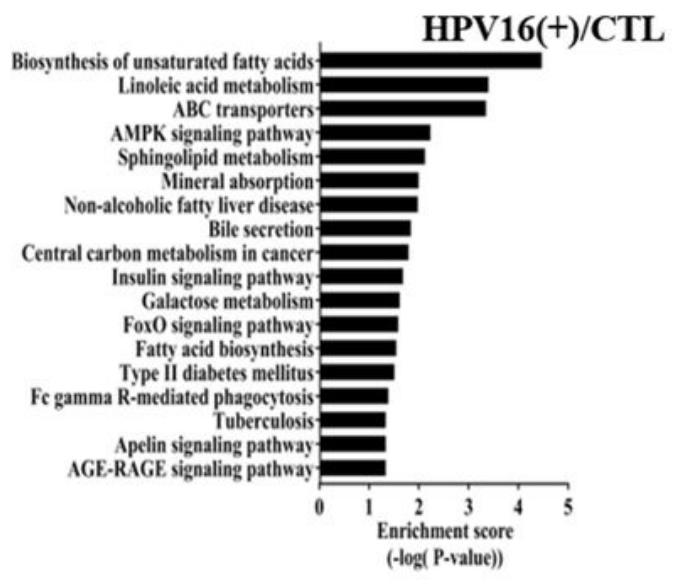

$\mathbf{E}$

HPV18(+)/CTL HPV16(+)/CTL

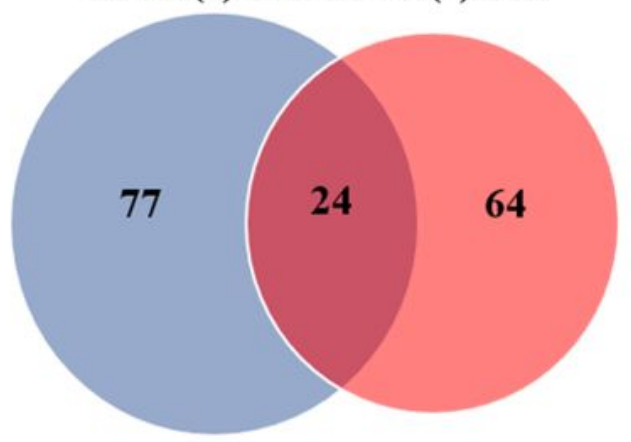

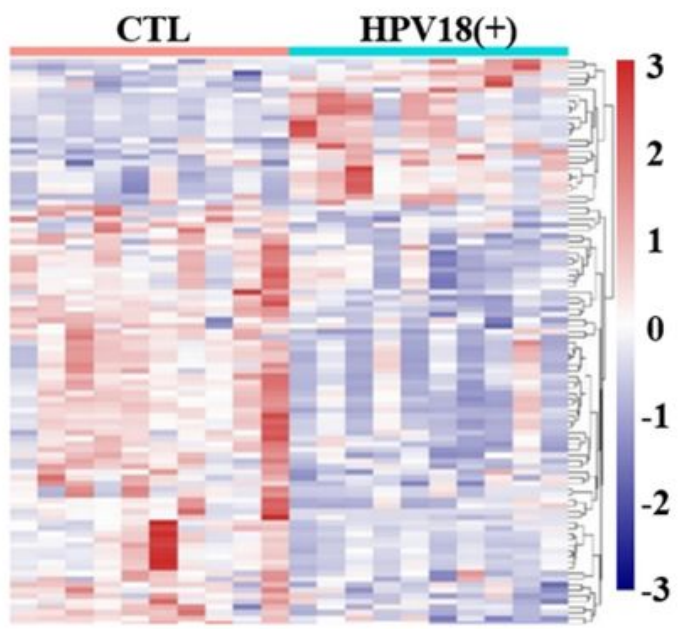

D

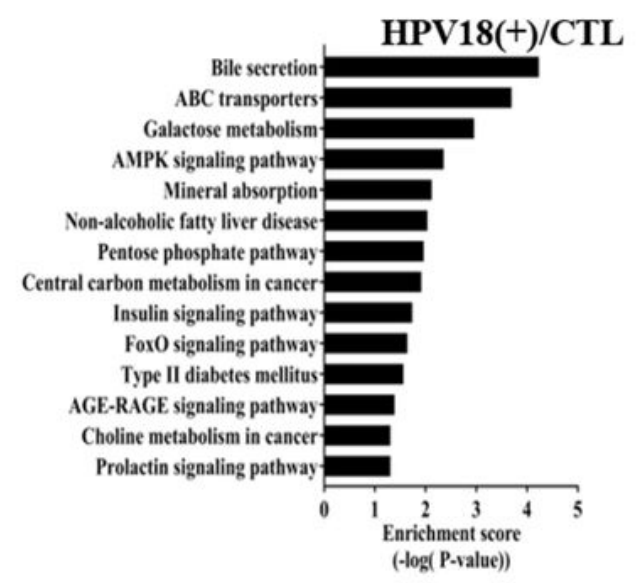

$\mathbf{F}$

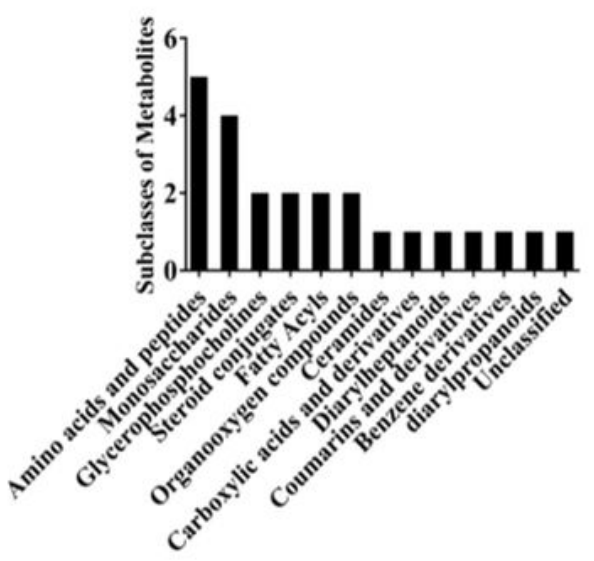

\section{Figure 2}

Analysis of HRHPV infection plasma reveals HPV-related differences of metabolites. A. Heatmap of differential metabolites with intensities determined by LC-MS. Hierarchical clustering analysis was used to assess significantly up-regulated and downregulated metabolites between HPV16 (+) and CTL plasma. B. Hierarchical clustering analysis was used to assess significantly up regulated and downregulated metabolites between HPV18 (+) and CTL plasma. Increased and decreased metabolite levels are depicted 
by red and blue colors, respectively. VIP, variable importance in projection.C. Pathway clustering analysis of HPV16 (+) samples, significantly enriched pathway clusters were identified based on p-values $<0.05$ and depicted in logarithmic scale (log2). D. Pathway clustering for metabolites enriched in HPV18 (+) samples by metabolomics analysis. E. Number of shared metabolites among the high-risk positive groups visualized on a Venn diagram. F. Number of shared metabolites that belong to glycerophosphocholines, amino acids and peptides, monosaccharides classes, etc.

A
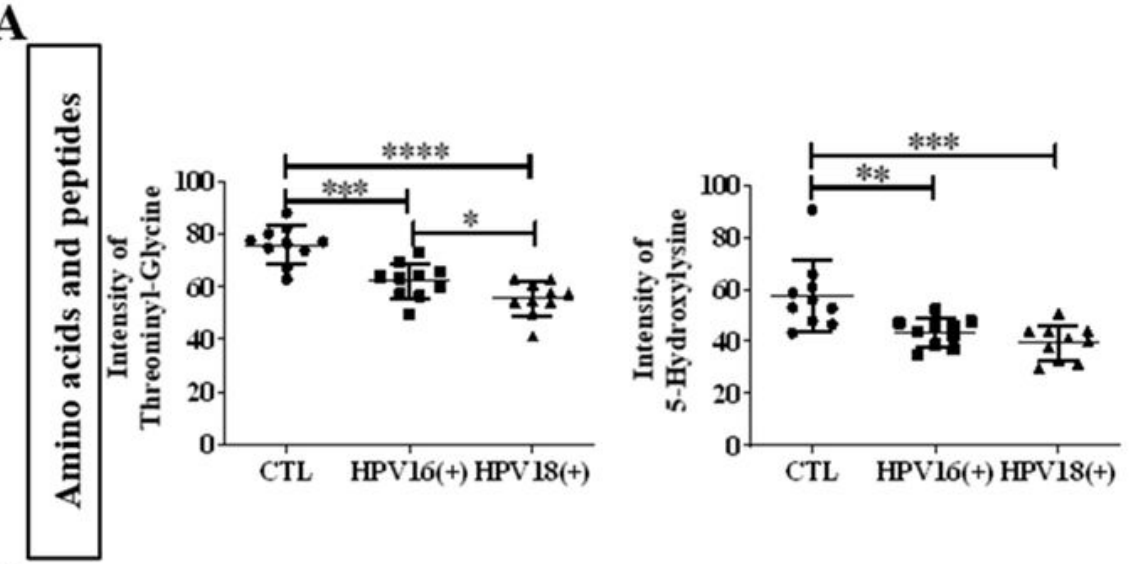

B
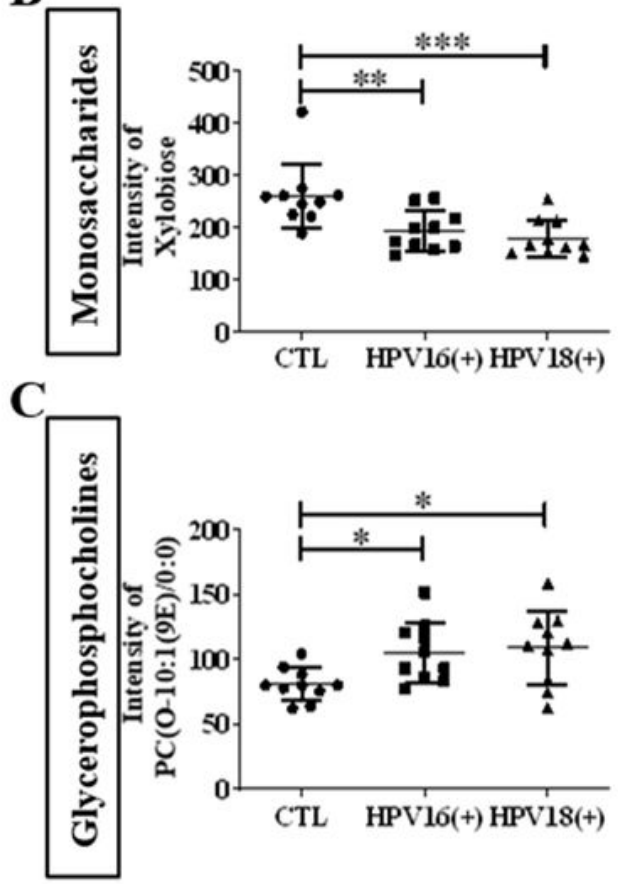

D
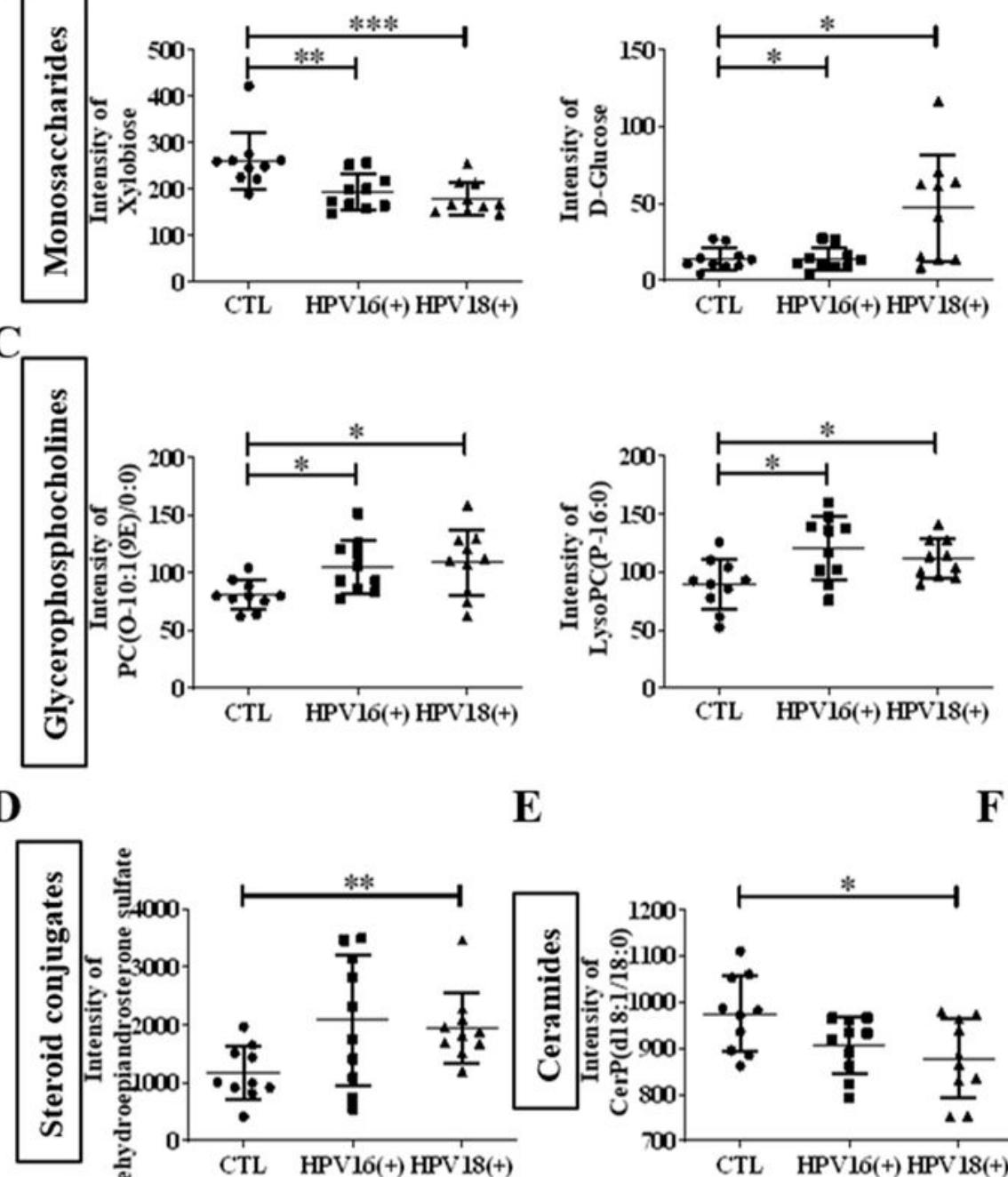

$\mathbf{E}$
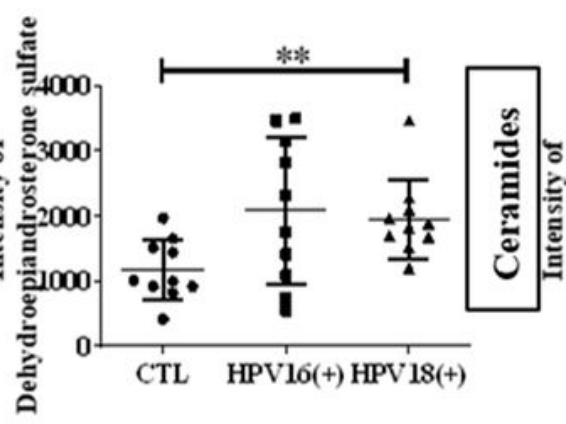
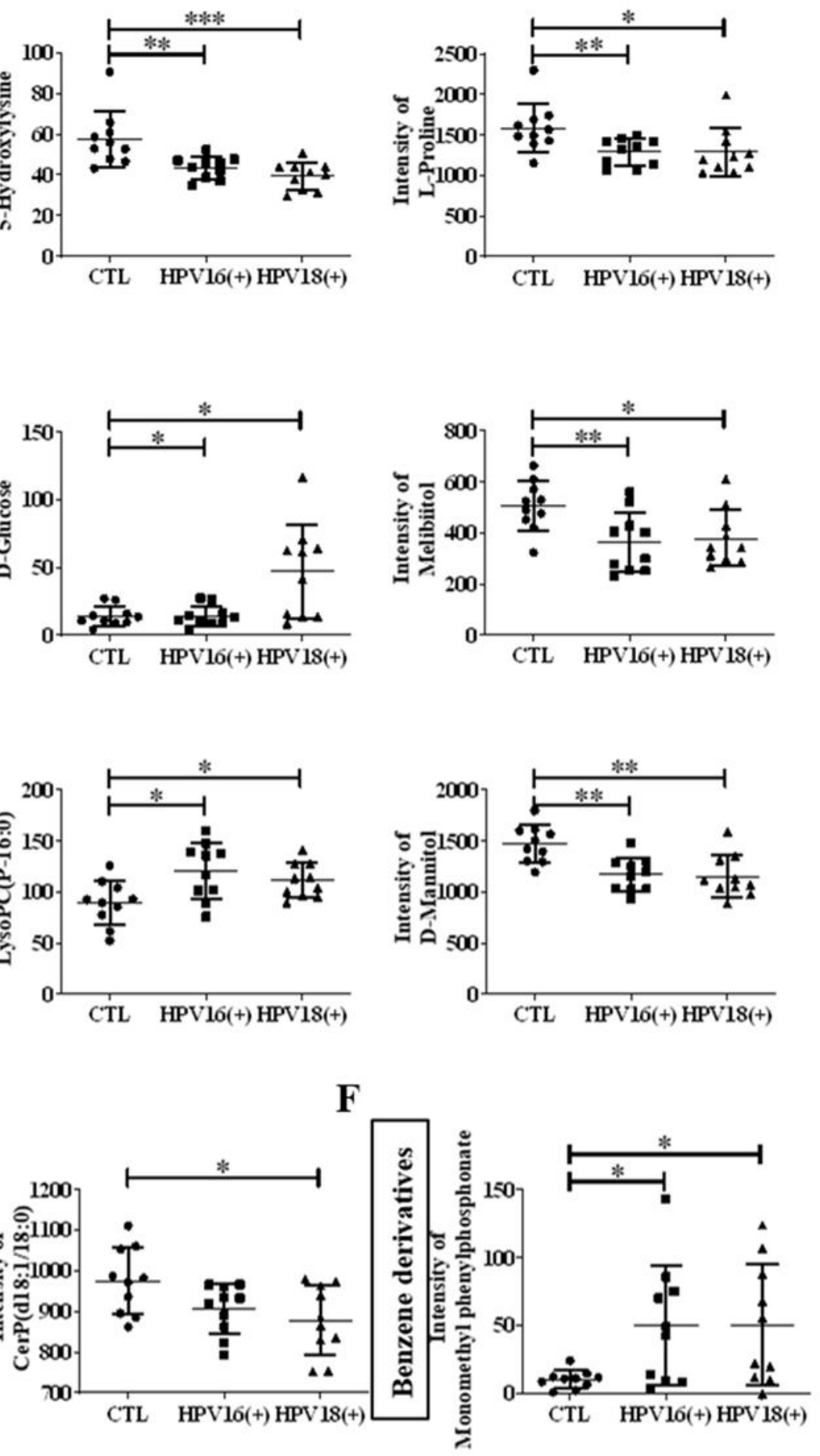

Figure 3 
Differences in the intensity of significantly different metabolites species between CTL and HRHPV infection groups. A. Amino acids and peptides were reduced in HRHPV groups. B. Monosaccharides were significantly reduced in HRHPV groups compared to CTL group. C. Glycerophosphocholines were enriched in HRHPV groups and. D-Mannitol was decreased in HRHPV groups. D-F. Steroid conjugates and benzene derivatives were increased, while ceramides were reduced in HRHPV groups
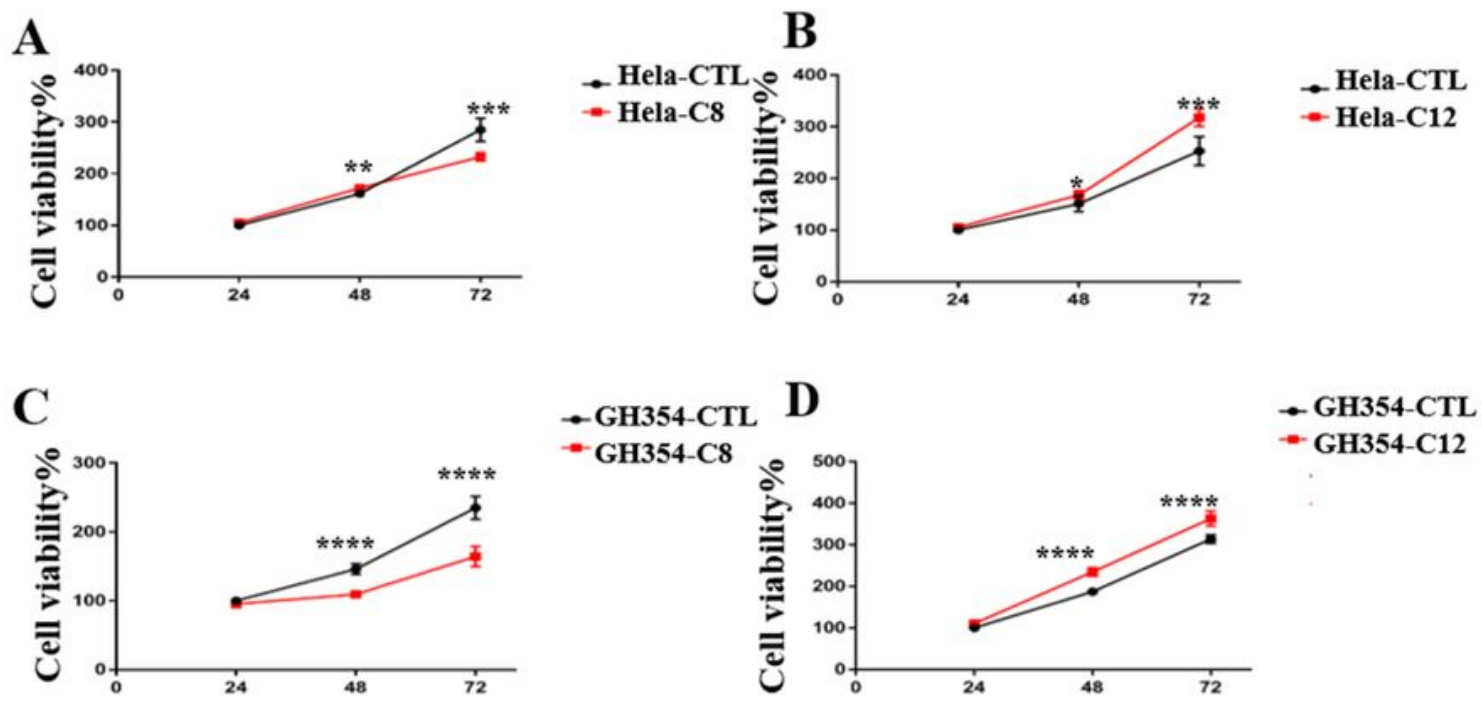

$\rightarrow$ GH354-CTL

- GH354-C12
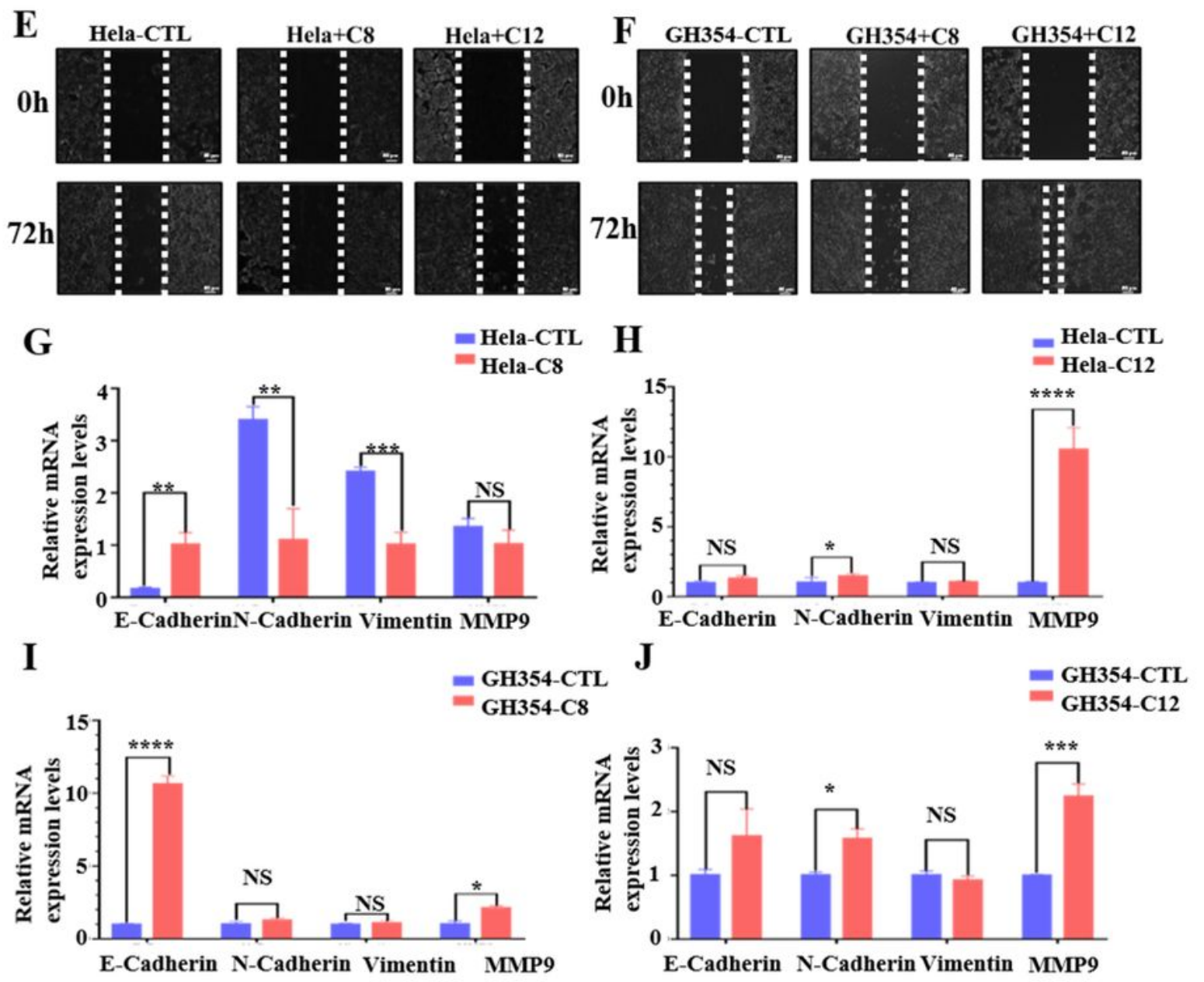

Figure 4 
C8 and C12 Altered proliferation and migration of CC cell lines and malignant behavior related molecules at mRNA levels A, C. C8 inhibited the proliferation of Hela and GH354 cells. B, D. C12 promoted the proliferation of Hela and GH354 cells. E, F. C8 inhibited cervical cancer cells migration while C12 promoted cervical cancer cells migration. G, I. Q PCR analysis the mRNA expression levels of E-cadherin, $\mathrm{N}$-cadherin, Vimentin and MMP9 under $\mathrm{C} 8$ intervention in HeLa and GH354 cells H, J. Q PCR analysis the mRNA expression levels of E-cadherin, N-cadherin, Vimentin and MMP9 under $\mathrm{C} 12$ intervention in HeLa and GH354 cells. Images are representative of 3 biological replicates. All values represent the mean \pm standard deviation (SD). A Scale bar of $50 \mu \mathrm{m}$ is shown. $\mathrm{n}=3$. ${ }^{*} \mathrm{P}<0.05$; $* * \mathrm{P}<0.01$; ${ }^{* \star *} \mathrm{P}<0.001$ 

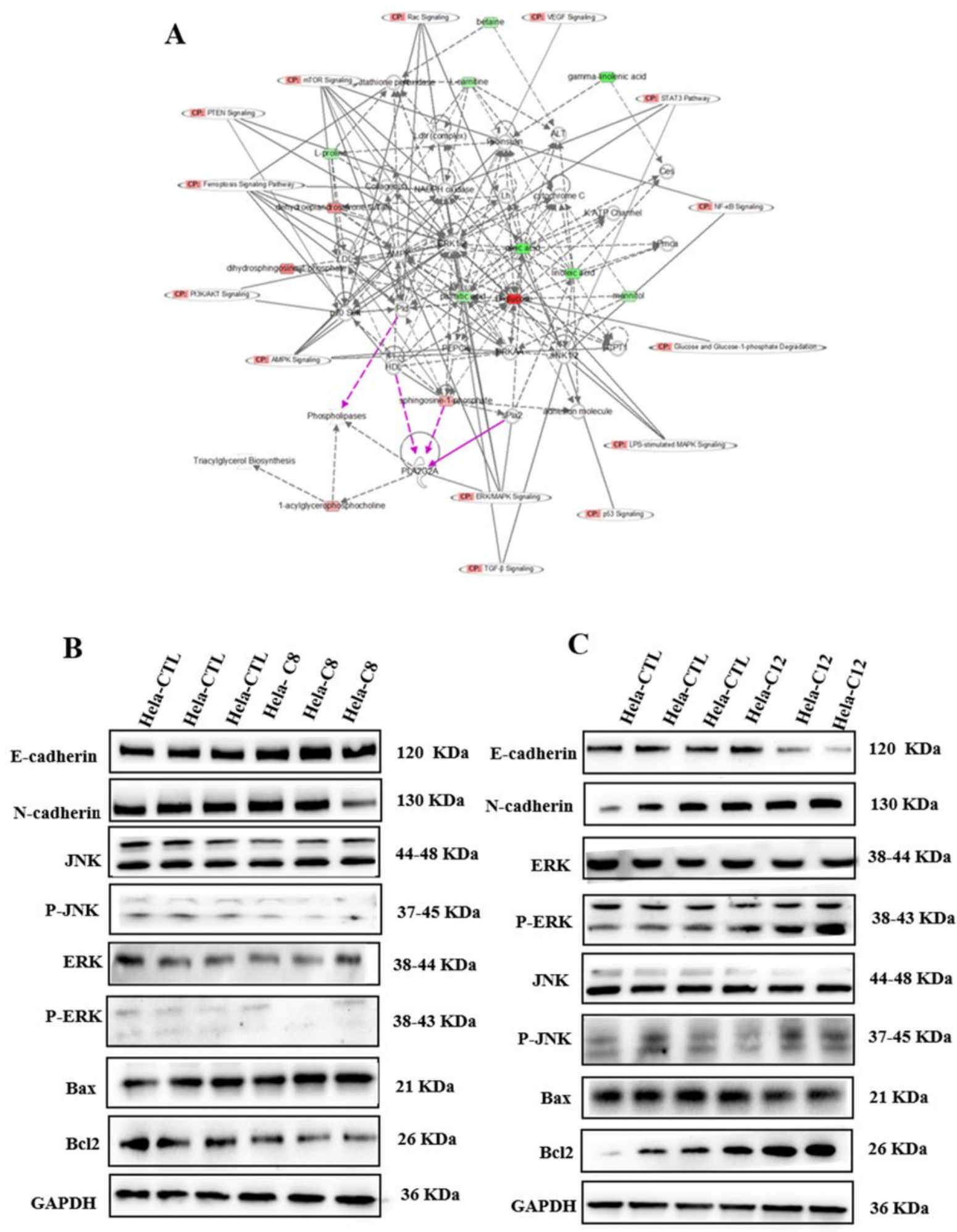

\section{Figure 5}

C8 and C12 altered malignant behavior related molecules and MAPK Signaling in Cervical Cancer Cells A. IPA analysis of metabolites related to biological network and canonical pathways and functions. Dysregulated metabolites were closely associated with PI3K/AKT signaling, mTOR signaling and PTEN signaling, and specific lipids were associated with ERK/MAPK signaling, TGF- $\beta$ signaling and PLA2G2A regulation. Lines represent the biological relationship between two nodes. Red symbols represent up- 
regulated metabolites; green symbols represent downregulated metabolites. B-C. Western blotting analysis of E-cadherin, N-cadherin, JNK, P JNK, ERK, P ERK, Bax and Blc2 protein levels in HeLa, HeLa/C12 and HeLa/C8 cells. ${ }^{*} \mathrm{P}<0.05 ;{ }^{* *} \mathrm{P}<0.01 ; * \star * \mathrm{P}<0.001, \mathrm{n}=3$ 